\title{
PREVALÊNCIA DO TABAGISMO E GRAU DE DEPENDÊNCIA ENTRE USUÁRIOS DO CENTRO DE ATENÇÃO À SAÚDE (CAS) - UVARANAS, PONTA GROSSA-PR
}

\author{
PREVALENCE OF SMOKING AND DEGREE OF ADDICTION AMONG \\ PATIENTS OF THE HEALTH CARE CENTER - UVARANAS, \\ PONTA GROSSA-PR
}

\author{
Andriele Costa Belinski ${ }^{1}$, Erildo Vicente Müller ${ }^{2}$ \\ ${ }^{1}$ Aluna do Curso de Especialização em Saúde Coletiva, Gestão de Saúde Pública, \\ Universidade Estadual de Ponta Grossa (UEPG). Email: andri_belinski@yahoo.com.br \\ ${ }^{2}$ Docente mestre do departamento de Enfermagem e Saúde Pública da Universidade \\ Estadual de Ponta Grossa (UEPG). E-mail: erildomuller@uepg.br
}

Recebido para publicação em 01/05/2010

Aceito para publicação em 14/06/2010

\section{RESUMO}

O tabagismo é considerado um relevante problema de Saúde Pública em todo o mundo. O hábito de fumar é a principal causa evitável de mortalidade, pois está associado a mortes prematuras e incapacidades devido a doenças cardiovasculares, respiratórias e neoplasias. Este estudo teve como objetivo conhecer a prevalência do tabagismo e o grau de dependência nicotínica entre usuários do Centro de Atenção à Saúde (CAS) - Uvaranas, Ponta Grossa-PR. Estudou-se uma amostra de 150 usuários, idade igual ou superior a 18 anos, sendo utilizado para coleta de dados um questionário com questões referentes a gênero, idade, escolaridade, renda mensal, hábito de fumar e interesse em abandonar o tabagismo, além do Teste de Fagerström para Dependência de Nicotina para mensurar a intensidade da dependência dessa substância. A prevalência de tabagismo foi de $19,3 \%$, sendo maior entre mulheres $(62,1 \%)$. O perfil dos fumantes apresentou maior concentração na faixa etária de adultos jovens $(34,5 \%)$, com 0 a 8 anos de estudo $(58,6 \%)$ e com renda familiar entre 2 a 5 salários mínimos $(62,1 \%)$. A pontuação média dos fumantes avaliados foi de baixa dependência $(3,4 \pm 2,6)$; desses, sete $(24,0 \%)$ foram classificados com dependência elevada ou muito elevada e $79,3 \%$ responderam estar motivados a parar de fumar, sendo indicado contato para inserção no tratamento do tabagismo.

Palavras-chave: Tabagismo. Prevalência. Dependência.

\begin{abstract}
Smoking is considered a relevant public health problem around the world. Smoking habit is the major preventable cause of death as it is associated to premature death
\end{abstract}


and disabilities due to cardiovascular diseases, breathing disorders and neoplasm. The aim of this study was to know the prevalence of smoking and the degree of nicotine dependence in the patients of the Health Care Center (Centro de Atenção à Saúde - CAS) - Uvaranas, Ponta Grossa - PR. The sample studied was of 150 (a hundred and fifty) patients of 18 years old or more. A questionnaire was used to collect data that included information about patients' gender, age, schooling, monthly income, smoking habits and interest in quitting smoking. In addition to the questionnaire patients took the Fagerström test to check their level of nicotine addiction. The prevalence of smoking was 19,3\%; among women the percentage was of $62,1 \%$. The profile of smokers shows a higher number in young adults $(34,5 \%)$, patients who had studied up to 8 years $(58,6 \%)$ and with a monthly income from 2 to 5 minimum wages $(62,1 \%)$ per family. The average score of the smokers studied demonstrated a low level of addiction $(3,4 \pm 2,6)$; of these $7(24 \%)$ were diagnosed with a high or very high level of addiction and $79,3 \%$ said they were motivated to quit smoking. These patients were suggested to take part in the anti-smoking program.

Keywords: Smoking. Prevalence. Addiction.

\section{Introdução}

O tabaco, obtido da planta Nicotiana tabacum L (Solanaceae), teve seu uso originário por indígenas, nas Américas, em cerimônias religiosas e com finalidade medicinal, sob diferentes formas: fumo, cataplasma, infusões, pois se acreditava em suas propriedades na cura de doenças, como bronquite crônica, asma, doenças do fígado e dos intestinos, reumatismo e outras. A partir disso, o uso se tornou progressivo a nível mundial, mesmo sob contestações de sua propriedade curativa (BRASIL, 2001).

Muitas formas de preparo do tabaco foram desenvolvidas e são utilizadas para consumo, como cigarro, charuto, cachimbo, narquilé, uso oral de tabaco (tabaco sem fumaça, moído, mascado) e rapé (BRASIL, 2009).

$\mathrm{O}$ crescimento do consumo de cigarro foi evidenciado principalmente no início do século XX, com a industrialização da produção de cigarros em larga escala e altos investimentos em propaganda e marketing. Essa pressão foi determinante, pois conduziu o hábito de fumar a associação de status, beleza, sucesso, liberdade, charme, o que tornou o cigarro objeto de desejo (PAN, 1992).

De acordo com estimativas mundiais, aproximadamente um terço da população adulta é fumante, o que implica em prejuízo à saúde, uma vez que o tabagismo é caracterizado como um dos principais fatores de risco para diversas doenças, como doenças cardiovasculares, neoplasias e as doenças respiratórias obstrutivas crônicas (BRASIL, 2004).

A fumaça decorrente da queima do tabaco apresenta aproximadamente 4720 substâncias químicas voláteis e material particulado(BRASIL, 2008) produzidas pela volatilização, pirólise e pirossíntese do tabaco (BURNS, 2008). Dentre essas, as mais importantes são o monóxido de carbono ( $\mathrm{CO}$ ), a nicotina e o alcatrão. Esses componentes se depositam nas vias respiratórias e superfície dos alvéolos pulmonares e desencadeiam ações farmacológicas, antigênicas, mutagênicas e carcinogênicas (HORTENSE; CARMAGNANI; BRÊTAS, 2008).

O uso do tabaco resulta em altas taxas de morbimortalidade, sendo a este atribuídas 40 a 45\% das mortes por câncer, 90 a 95\% de mortes por câncer de pulmão, $75 \%$ das mortes por Doença Pulmonar Obstrutiva Crônica (DPOC), 35\% das mortes por doenças cardiovasculares, em homens com idade de 35 a 69 anos em países desenvolvidos (WHO, 1999).

O tabagismo produz, além disso, impacto socioeconômico e ambiental (BECKER et al., 2005). Em relação aos efeitos socioeconômicos estão a redução de gastos com necessidades básicas, queda na produtividade (absenteísmo no trabalho, acidentes, assistência médica, invalidez, mortes precoces). 
Quanto ao prejuízo ambiental, o cultivo do tabaco leva ao empobrecimento do solo, à poluição por pesticidas e fertilizantes e ao desmatamento dessas áreas (BRASIL, 2009).

O Brasil é o segundo maior produtor e o maior exportador mundial de folhas de fumo. A Região Sul é a que apresenta a maior produção, com percentual de $97 \%$, liderada pelo Rio Grande do Sul, com 50\%, estando Santa Catarina na segunda posição, seguido pelo Paraná (CAVALCANTE, 2005).

Atualmente, o tabagismo está caracterizado como uma doença epidêmica resultante da dependência de nicotina e é classificado, desde 1993, pela Organização Mundial de Saúde (OMS) dentro do grupo de transtornos mentais e de comportamento decorrente do uso de substâncias psicoativas na Décima Revisão da Classificação Internacional de Doenças (CID-10). (OMS, 1997).

Nessa perspectiva, a OMS atribui ao consumo de tabaco a principal causa de morte evitável no mundo, e o caracteriza como problema de saúde pública tanto em países desenvolvidos como em desenvolvimento(WHO, 2008).

O tabagismo passivo é denominado o ato de inalação involuntária da fumaça decorrente da queima de tabaco e, de acordo com a OMS, é considerado a terceira maior causa de morte que poderia ser evitada no mundo, subsequente ao tabagismo ativo e ao consumo excessivo de álcool (REICHERT et al., 2008).

O número de mortes por ano relacionadas ao uso do tabaco é de 4,9 milhões, o que representa mais de 10 mil mortes por dia. Haverá aumento para 10 milhões de mortes/ano em torno do ano 2020 se o consumo seguir a mesma expansão, atingindo, sobretudo, indivíduos em idade produtiva (BRASIL, 2003). Destas, $70 \%$ serão registradas em países em desenvolvimento e superam o número de óbitos devido ao HIV, malária, tuberculose, alcoolismo, causas maternas, homicídios e suicídios combinados (BRASIL, 2009).

Os fatores que influenciam o início do tabagismo estão associados, principalmente, a comportamentos, rituais, hábitos individuais e sociais que criam reflexos condicionados. Dentre esses, citam-se o meio de convívio do indivíduo, visualização do cigarro como atrativo pessoal, símbolo de força, alívio das tensões e da insegurança, além da curiosidade em experimentar (HORTENSE; CARMAGNANI; BRÊTAS, 2008). Soma-se a estes a facilidade de acesso físico e o baixo custo do cigarro, o qual é influenciado pelo intenso mercado ilegal a que se atribui em torno de $35 \%$ do consumo nacional (BRASIL, 2003).

Aproxidamente 80 a $90 \%$ dos fumantes iniciam o hábito antes dos 18 anos de idade, sendo que nos países em desenvolvimento a maior parte dos casos ocorre em torno dos 12 anos (BRASIL, 2004). O início do uso do tabaco na adolescência tem como fatores associados pais tabagistas, colegas mais velhos e propaganda intensa da mídia (MARQUES; RIBEIRO, 2002).

Ao iniciar o uso de cigarros são relatados efeitos indesejados como dor de cabeça, insônia, náuseas, tontura, nervosismo, tosse, os quais são atenuados pelo uso contínuo, o que é denominado tolerância, que se apresenta como necessidade de doses cada vez maiores da substância para manter o nível de satisfação inicial, culminando ao padrão de consumo diário do tabagista(MARQUES; RIBEIRO, 2002). O resultado disso é um quadro crônico, o que eleva o risco de desenvolvimento de doenças debilitantes (IGLESIAS et al., 2007).

A nicotina, segundo estudos clínicos, é a substância ativa responsável pelo desenvolvimento da dependência (PLANETA; CRUZ, 2005), uma vez que é rapidamente absorvida pelos alvéolos pulmonares e atinge o cérebro em aproximadamente 10 segundos (BALBANI; MONTOVANI, 2005).

Adependência de nicotina, assim como ocorre com outras drogas de abuso, envolve o efeito reforçador positivo e de sensibilização comportamental através de suas ações agudas no sistema dopaminérgico mesolímbico ou por neuroadaptações desse sistema, em consequência da exposição prolongada à droga (HALTY et al., 2002).

A ação psicoativa de estímulo da sensação de prazer é mediada pela dopamina (HORTENSE; CARMAGNANI; BRÊTAS, 2008), que também controla o impulso motivacional e estímulos reforçadores no sistema nervoso central. Através da sensibilização esses estímulos, consumo da droga e comportamentos relacionados direcionam ao 
aumento da necessidade, o que induz a persistência do tabagismo e a dificuldade em sua suspensão.

A redução em $50 \%$ no consumo de nicotina desencadeia a síndrome de abstinência, que se caracteriza por um conjunto de sinais e sintomas, em geral, contrários aos efeitos agudos da droga, e resulta em desconforto intenso ao dependente, o que é atribuído por dependência física (HALTY et al., 2002). O principal sintoma é fissura, que corresponde ao desejo intenso de fumar e cede em torno de cinco minutos (REICHERT et al., 2008).

O quadro inicia aproximadamente 8 horas após o último cigarro e atinge o pico no terceiro dia, desencadeando ansiedade, irritabilidade, distúrbios do sono (insônia e sonolência diurna), aumento do apetite, problemas de concentração e atenção (HALTY et al., 2002). Em vista disso, o dependente sente-se aliviado ao fumar o primeiro cigarro da manhã (BALBANI; MONTOVANI, 2005).

O tratamento do dependente de tabaco relaciona intervenções comportamentais, terapia cognitivo-comportamental, entre aconselhamento apoio psicológico, e farmacológicas, com administração de medicamentos, que têm como finalidade a redução ou superação da dependência (HALTY et al., 2002). A abordagem do fumante pode ser mínima, com breve aconselhamento de até dez minutos, com enfoque na motivação de mudanças de comportamento; ou intensiva, de aproximadamente 90 minutos, individual ou em grupo, em que ocorre o preparo para o fumante resistir à vontade de fumar, prevenir recaídas e enfrentar situações de tensão (CARVALHO, 2009).

Há dois métodos que podem ser utilizados para deixar de fumar: parada abrupta, em que o fumante cessa totalmente, e parada gradual que ocorre em alguns dias, sendo recomendado para aqueles com intensa ansiedade. Essa última é dividida em duas formas: redução e adiamento. Na primeira, o fumante reduz-se o número de cigarros fumado a cada dia, enquanto na segunda é adiada a hora em que se inicia o ato de fumar, para que esse ocorra cada dia mais tarde. Na prática, verifica-se como melhor método a parada abrupta (BRASIL, 2008).

A farmacoterapia indicada para os dependentes da nicotina divide-se em fármacos de primeira linha (bupropiona e terapia de reposição da nicotina) e de segunda linha (clonidina e nortriptilina). O tratamento apresenta eficácia aumentada com a participação em grupos de autoajuda e psicoterapia em sessões de aconselhamento, sobretudo quando o paciente apresenta quadro depressivo e ansiedade (BALBANI; MONTOVANI, 2005).

$\mathrm{O}$ aconselhamento é importante para o tabagista como forma de aprender estratégias a fim de romper o vínculo entre determinadas situações e a busca automática pelo cigarro, como comportamento associativo (após as refeições, ao tomar café ou reunião com amigos) ou momentos de estresse, nervosismo, angústia(BALBANI; MONTOVANI, 2005).

A cessação do hábito de fumar promove benefícios em qualquer idade, pois reduz significativamente o risco de doenças decorrentes do consumo a curto e médio prazo (HALTY et al., 2002), o que diminui a morbimortalidade e melhora a condição geral de saúde contribuindo para aumento na qualidade de vida do indivíduo (CARVALHO, 2010).

Diante do panorama acima exposto, este trabalho teve como objetivo conhecer a prevalência do tabagismo e o grau de dependência nicotínica entre usuários do Centro de Atenção à Saúde (CAS) Uvaranas, Ponta Grossa-PR, e verificar o interesse desses fumantes quanto ao abandono do tabagismo.

\section{Materiais e métodos}

Trata-se de um estudo observacional, de delineamento transversal, executado no Centro de Atenção à Saúde (CAS) - Uvaranas, município de Ponta Grossa-PR. A amostra foi selecionada entre usuários, idade igual ou superior a 18 anos, no mês de agosto de 2010.

A coleta de dados para determinação da prevalência do tabagismo entre os usuários do serviço foi obtida através da aplicação de um questionário estruturado constituído de questões abertas e fechadas referentes à identificação, gênero, idade, renda familiar, escolaridade, hábito de fumar (fumante, ex-fumante, nunca fumou), há quanto tempo fumava, além do interesse, se fumante, em abandonar o tabagismo (Anexo 1). 
Caracterizou-se como fumante o indivíduo que utilizou o tabaco diariamente, nos últimos seis meses, e, não fumante aquele que não fez uso de tabaco nos últimos seis meses anteriores a pesquisa.

A fim de avaliar o grau de dependência da nicotina entre os fumantes, foi aplicado o Fagerström Test for Nicotine Dependence (FTND, Teste de Fagerström para Dependência de Nicotina), criado em 1978 por Fagerström et al. (FAGERSTROM, 1978), com originais em inglês e sueco, adaptado a várias línguas (Anexo 2). O FTND foi inicialmente desenvolvido para verificar a necessidade de terapia de reposição de nicotina no tratamento do fumante, visto que uma soma acima de 6 pontos é indicativa de que o paciente apresentará desconforto (síndrome de abstinência) ao deixar de fumar.

O FTND constitui-se de seis questões, sendo atribuída a cada resposta um valor. A soma dos pontos ao final do questionário resulta num escore, que varia de 0 a 10, e indica o grau de dependência do fumante em: muito baixo ( 0 a 2 pontos), baixo ( 3 a 4 pontos), médio (5), elevado ( 6 a 7 ), muito elevado (8 a 10). (BALBANI; MONTOVANI, 2005).

Os questionários foram previamente testados em estudo piloto com um grupo de pessoas que não integravam a amostra do estudo, com intuito de verificar o nível de compreensão das perguntas e avaliar a necessidade de realizar alterações antes da aplicação na amostra.

Em princípio, os usuários eram esclarecidos em relação às condições da pesquisa, como objetivos, justificativa e possíveis benefícios decorrentes da mesma, e faziam a leitura do Termo de Consentimento Livre e Esclarecido (TCLE). Aqueles que concordaram em participar da pesquisa assinaram o TCLE, sendo garantida aos mesmos a confidencialidade e sigilo dos dados fornecidos.

A amostragem foi não probabilística, selecionada aleatoriamente, entre os usuários que procuravam atendimento no local. $\mathrm{O}$ número de questionários válidos aplicados foi de 150 .

Os dados coletados foram analisados mediante estatística descritiva, sendo as variáveis categóricas expressas em frequência por número e percentual e as variáveis contínuas através de medidas de tendência central (média).
O contato foi aproveitado para motivar os fumantes que tivessem interesse, à inserção no tratamento do tabagismo junto ao programa de extensão Educando e Tratando o Tabagismo na UEPG.

A presente pesquisa foi submetida e aprovada pelo Comitê de Ética e Pesquisa em Seres Humanos da Universidade Estadual de Ponta Grossa (COEP), sob protocolo número 44/2010.

\section{Resultados}

O questionário foi aplicado a 150 usuários do CAS-Uvaranas, $50(33,3 \%)$ do gênero masculino e $100(66,7 \%)$ do gênero feminino. A média de idade foi de 37,9 $\pm 14,3$ anos, com idade mínima de 18 e máxima de 84 anos. Quanto à escolaridade, 80 $(53,3 \%)$ tinham de 0 a 8 anos de estudo e $70(46,7 \%)$ haviam estudado por mais de oito anos. Em relação à situação socioeconômica, 51 (34,0\%) usuários possuíam renda familiar inferior a dois salários mínimos; $93(62,0 \%)$ de 2 a 5 salários e seis $(4,0 \%)$ tinham renda superior a cinco salários mínimos.

Quando questionados quanto ao hábito de fumar, 37 usuários $(24,7 \%)$ afirmaram ser ex-fumantes e $84(56,0 \%)$ relataram nunca terem fumado anteriormente.

A prevalência de fumantes obtida para a amostra em estudo foi de $19,3 \%$, dos quais $37,9 \%$ eram homens e $62,1 \%$ do gênero feminino. O perfil dos fumantes em relação à idade apresentou maior concentração nas faixas etárias de adultos jovens e idosos (Figura 1). 
Figura 1 - Perfil dos fumantes quanto à idade

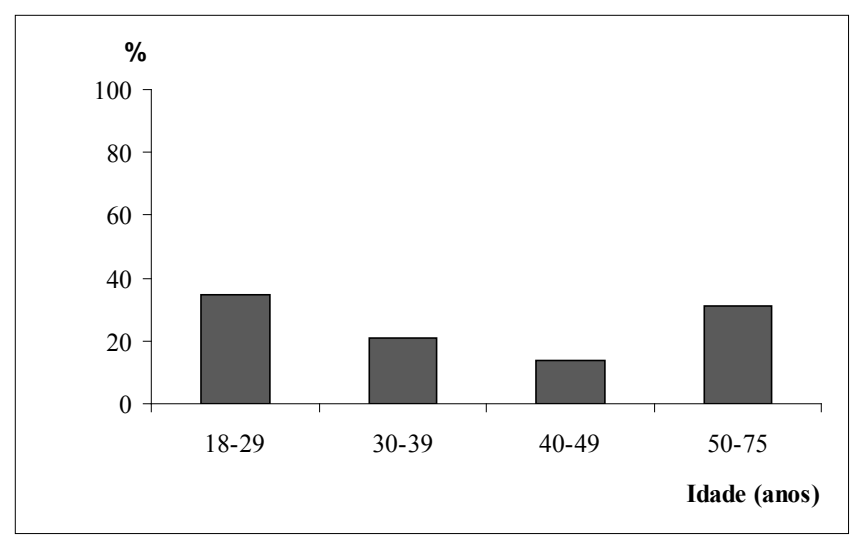

Fonte: Pesquisa de Campo

Quanto à prevalência de tabagismo pela escolaridade, observa-se, através da Tabela 1, que a maior parte dos fumantes possuía de 0 a 8 anos de estudo.

Tabela 1 - Perfil dos fumantes em relação à escolaridade

\begin{tabular}{ccc}
\hline Variáveis & $\mathrm{N}$ & $\%$ \\
\hline Escolaridade (anos de estudo) & & \\
- $\quad 0$ a 8 anos & 17 & 58,6 \\
- $\quad$ mais de 8 anos & 12 & 41,4 \\
- TOTAL & 29 & 100 \\
\hline
\end{tabular}

Fonte: Pesquisa de Campo

Houve ainda variação do número de fumantes de acordo com a renda familiar (em salários mínimos), sendo principalmente de 2 a 5 salários mínimos, conforme a Tabela 2.

Tabela 2 - Perfil dos fumantes em relação à renda

\begin{tabular}{llcc}
\hline & Variáveis & $\mathrm{N}$ & $\%$ \\
\hline & Renda & & \\
• & $<2$ salários-mínimos & 10 & 34,5 \\
- & $2-5$ salários-mínimos & 18 & 62,1 \\
- & $>5$ salários-mínimos & 1 & 3,4 \\
• & TOTAL & 29 & 100 \\
\hline
\end{tabular}

Fonte: Pesquisa de Campo

Em relação à questão que indagava há quanto tempo o fumante fazia uso do cigarro, 20 fumantes $(69,0 \%)$ responderam fumar há mais de 10 anos e nove $(31,0 \%)$ disseram que usavam o cigarro há menos de 10 anos.

A partir da soma de pontos obtida com as respostas referentes à aplicação do FTND, o grau de dependência nicotínica foi classificado em cinco graus: muito baixo, baixo, médio, elevado e muito elevado (Tabela 3). A amostra em estudo apresentou um valor médio de dependência de 3,4 $\pm 2,6$. Dos 29 fumantes avaliados, sete $(24,0 \%)$ foram classificados com grau de dependência elevado ou muito elevado.

Tabela 3 - Grau de dependência medido pelo Teste de Fagerström para Dependência de Nicotina

\begin{tabular}{llccc}
\hline Grau de dependência & Pontos & $\mathrm{N}$ & $\%$ \\
\hline - & Muito baixo & $0-2$ & 12 & 41,4 \\
- & Baixo & $3-4$ & 9 & 31,0 \\
- & Médio & 5 & 1 & 3,5 \\
- & Elevado & $6-7$ & 5 & 17,2 \\
- & Muito Elevado & $8-10$ & 2 & 6,9 \\
- & & 29 & 100 \\
\hline
\end{tabular}

Fonte: Pesquisa de campo

Nesta pesquisa foi considerado como ponto de corte entre fumantes de baixa e de elevada dependência o valor de seis pontos. Dessa forma, o grupo considerado de elevada dependência nicotínica (GEDN $=24,1 \%$ ) correspondeu aos fumantes que tiveram seis ou mais pontos; e o grupo de baixa dependência nicotínica (GBDN $=75,9 \%$ ) somou menos que seis pontos (Tabela 4). Essa classificação, a partir da aplicação do FTND, permite avaliar a dificuldade que o fumante apresentará na conduta da cessação do tabagismo ao estar no GEDN devido ao desconforto significativo (síndrome de abstinência). (REICHERT et al., 2008) 
Tabela 4 - Gênero e grau de dependência nicotínica

\begin{tabular}{|c|c|c|c|}
\hline Gênero & GEDN & GBDN & Total \\
\hline - $\quad$ Feminino & $316,7 \%$ & $1583,3 \%$ & 18 \\
\hline - Masculino & $4 \quad 36,4 \%$ & $7 \quad 63,6 \%$ & 11 \\
\hline • $\quad$ TOTAL & $7 \quad 24,1 \%$ & $2275,9 \%$ & 29 \\
\hline
\end{tabular}

GEDN - Grupo de elevada dependência nicotínica ( $\geq 6$ pontos) GBDN - Grupo de baixa dependência nicotínica

Quanto à motivação, 23 (79,3\%) dos usuários fumantes responderam estar motivados a parar de fumar, enquanto seis $(20,7 \%)$ não apresentaram interesse no abandono do tabagismo. Desses que demonstraram interesse, foi indicado contato para inserção no tratamento do tabagismo junto ao programa de extensão Educando e Tratando o Tabagismo na UEPG.

\section{Discussão}

O tabagismo é associado a muitos problemas de saúde, que acometem fumantes ativos e passivos, tendo como consequência um elevado impacto econômico para a sociedade, devido à demanda por assistência médica e queda de produtividade decorrente da morbidade e mortes prematuras (COELHO et al., 2010).

No Brasil, ocorrem ao ano aproximadamente 200 mil óbitos associados ao tabagismo. Em 2005, o gasto total do SUS com as doenças relacionadas ao tabaco, incluindo internações e quimioterapia, foi de $\mathrm{R} \$ 338.692 .516,02$, sobretudo em indivíduos com mais de 35 anos (PINTO; UGÁ, 2010).

A Pesquisa Nacional de Saúde e Nutrição (PNSN), realizada em 1989, foi a primeira pesquisa de grande alcance para estimar o percentual de fumantes no Brasil antes da instituição do Programa Nacional de Controle do Tabagismo (PNCT). Naquele ano, a prevalência de fumantes foi de $34,6 \%$, considerando a população com idade superior a 18 anos. Em 2002/2003, foi realizada uma pesquisa semelhante abrangendo 15 capitais e o Distrito Federal, cujo resultado foi igual a $19 \%$ de fumantes na população com 15 anos ou mais (BRATS, 2010).
O percentual obtido neste estudo (19,3\% de fumantes) foi comparável ao descrito na literatura (FILHO et al., 2009), pois o Brasil apresentou queda na prevalência do tabagismo para aproximadamente $17,5 \%$ em 2008, o que poderia ser associado ao desenvolvimento de programas e implantação de fortes políticas de controle do tabaco, como campanhas na mídia sobre os malefícios do cigarro, advertências através de imagens impactantes e incentivo a ambientes livres da fumaça do tabaco (BRATS, 2010).

A Pesquisa Especial de Tabagismo (PETab) realizada em 2008, cuja finalidade era verificar o panorama referente ao uso de produtos derivados do tabaco no país, revelou o número de fumantes correspondente a 24,6 milhões de pessoas, sendo que em todas as regiões, o percentual de homens usuários era maior que o de mulheres (IBGE, 2009).

Isso não foi observado nos resultados deste estudo, em que houve maior número de mulheres fumantes, o que poderia ser explicado pelo fato de os sujeitos da pesquisa não terem se distribuído de forma homogênea quanto ao gênero, devido à maior procura das mulheres por atendimento nos serviços de saúde, seja para si mesmas ou para seus filhos. Porém, nos últimos anos, relata-se que esteja ocorrendo o aumento do tabagismo entre mulheres e redução na curva de homens fumantes (BORGES; BARBOSA, 2009).

Em relação às características socioeconômicas, a prevalência de fumantes foi mais elevada em indivíduos com nível de escolaridade mais baixo e menor renda familiar, mantendo a forte correlação do tabagismo a um comportamento de grupos menos favorecidos com menor acesso à informação, educação e assistência à saúde (BORGES; BARBOSA, 2009).

Os fumantes brasileiros consomem, em média 15,4 cigarros/dia; 91\% relatam ser dependentes do cigarro e $52 \%$ 'muito dependentes'. A maioria dos fumantes salienta preocupação com problemas de saúde decorrentes do cigarro e relata que o fumo já lhe acarretou prejuízo à saúde (PROJETO ITC, 2009).

A aplicação do FTND, utilizado como instrumento de medida do grau de dependência nicotínica, detectou um valor médio classificado como baixa 
dependência entre os usuários fumantes do CAS-Uvaranas. De acordo com a caracterização dos fumantes, observou-se que a maior parte está no grupo considerado de baixa dependência (GBDN $=75,9 \%$ ), o que permite afirmar que teriam menor dificuldade em parar de fumar, no caso de estes entrarem num programa de cessação do fumo.

$\mathrm{O}$ intuito deste resultado é direcionar o tratamento do tabagista, visto que quanto maior a pontuação, mais dependente e maior a dificuldade do fumante atingir êxito na suspensão do tabagismo, principalmente devido à síndrome de abstinência.

Observou-se que a maior parte dos fumantes demonstrou interesse e motivação para parar de fumar, no entanto, esses necessitam de apoio/aconselhamento, pois o tabagista requer, em média, três a cinco tentativas até que consiga interromper o 'vício' definitivamente. Isso porque o período de maior risco de recaída é no primeiro ano de abstinência e ocorre, principalmente, devido a fatores emocionais (PRESMAN; CARNEIRO; GIGLIOTTI, 2005).

Apenas 5\% dos fumantes que desejam parar de fumar conseguem obter esse resultado. Aproximadamente $40 \%$ deles realizam tentativas sérias para controlar a dependência, contudo, $90 \%$ dessas ocorrem sem tratamento formal. Daqueles que tentam parar de fumar, 33\% conseguem manter abstinência por dois dias, e somente 3 a $5 \%$ o fazem por até um ano (MUNDIM; BUENO, 2006).

De acordo com a PETab, do total de fumantes avaliados, 33,5\% relataram que havia intenção de parar algum dia, contudo isso não ocorreria nos 12 meses seguintes, uma porcentagem de 11,4\% cogitavam parar nos 12 meses seguintes e 7,3\% tinham a intenção programada para o mês seguinte (IBGE, 2009).

O Ministério da Saúde, desde 1989, em vista da promoção de saúde, através do Instituto Nacional do Câncer (INCA), estabelece ações do Programa Nacional de Controle do Tabagismo (PNCT), cujo objetivo principal é reduzir a prevalência de fumantes e por consequência a morbimortalidade decorrente do consumo do tabaco. Para isso, faz-se necessário diminuir a iniciação do tabagismo, elevar o número dos que deixam de fumar e proporcionar proteção aos expostos ao tabagismo passivo (CAVALCANTE, 2005).
O Sistema Único de Saúde (SUS) disponibiliza o tratamento do tabagismo conforme a Portaria No 1035/GM de 31 de maio de 2004, regulamentada pela Portaria SAS/MS/No 442 de 13 de agosto de 2004. Essas portarias estabelecem a ampliação do acesso à abordagem e tratamento do tabagismo para atenção básica e média complexidade e incluem o Plano de Implantação da Abordagem e Tratamento do Tabagismo na Rede SUS (Anexo I da Portaria SAS/MS/No 442/2004) e o Protocolo Clínico e Diretrizes Terapêuticas da Dependência à Nicotina (Anexo II da Portaria SAS/MS/No 442/2004) (BRASIL, 2010).

O Plano de Implantação apresenta ênfase na capacitação de profissionais de saúde para inserir o tratamento em municípios com população superior a 100.000 habitantes, polos regionais e capitais. Para receber o material de apoio (Manual do Participante) para as sessões de abordagem e medicamentos, as unidades de saúde devem ser credenciadas no Cadastro Nacional de Estabelecimentos de Saúde (CNES) (CARVALHO, 2009).

A abordagem cognitivo-comportamental é obrigatória e a farmacoterapia, quando necessário, para todo fumante que tem interesse em parar de fumar e busca o tratamento em unidades de saúde do SUS cadastradas. O objetivo é auxiliar os participantes a deixarem de fumar e a permanecerem sem cigarros, através de informações em relação aos riscos do tabagismo, benefícios em parar de fumar e também estratégias de autocontrole para evitar o ciclo da dependência (BRASIL, 2001).

Em 2008, 465 municípios realizaram ações para o tratamento do tabagismo através do SUS, sendo a maior parte desses, concentrada na Região Sul - Rio Grande do Sul (136), Paraná (76) e Santa Catarina (51) (CARVALHO, 2009).

\section{Conclusão}

O tabagista é caracterizado como portador de um quadro crônico com necessidade de intervenção, seja através de aconselhamento psicológico, individual ou em grupo, associado ou não a terapia medicamentosa, que deve ser disponibilizada pelo SUS. 
A maioria dos indivíduos inicia o hábito de fumar na adolescência, o que indica a necessidade de instituir medidas preventivas nessa faixa etária, através de ações educativas nas instituições de ensino e assistência médica efetiva, com enfoque nos malefícios provenientes do uso do tabaco.

A aplicação do FTND é um método simples, rápido e de baixo custo, o qual permite identificar o grau de dependência nicotínica que se correlaciona ao desconforto no momento de deixar de fumar e a necessidade da forma de abordagem no tratamento para o controle dos sintomas de abstinência.

Parar de fumar envolve mudança no estilo de vida e proporciona benefícios à saúde, sendo o desejo de muitos fumantes, que por vezes ficam sem abordagem por parte dos profissionais de saúde. Um dos obstáculos enfrentados é a falta de tempo durante a consulta médica e a elevada demanda dos serviços de saúde.

É essencial que o paciente se sinta motivado a interromper o tabagismo desde o início e durante o tratamento. Em vista disso, uma ação com relevantes resultados sobre a redução da prevalência do tabagismo seria a introdução do aconselhamento mínimo nos serviços de atenção primária de saúde.

\section{REFERÊNCIAS}

BALBANI, A. P. S.; MONTOVANI, J. C. Métodos para o abandono do tabagismo. Rev Bras Otorrinolaringol., São Paulo, n. 71, p. 820-27, nov./dez. 2005.

BECKER, I. C. et al.. Prevalência do tabagismo na população urbana de Tubarão-SC. ACM Arq. Catarin. Med., Florianópolis, v. 34, n. 3, p. 37-44, 2005.

BOLETIM BRASILEIRO DE AVALIAÇÃO DE TECNOLOGIAS EM SAÚDE (BRATS). Estratégias clínicas para a cessação do tabagismo. Brasília: ANVISA, 2010.

BORGES, M. T. T.; BARBOSA, R. H. S. As marcas de gênero no fumar feminino: uma aproximação sociológica do tabagismo em mulheres. Ciênc. Saúde Coletiva., Rio de Janeiro, v. 14, n. 4, p. 1129-39, jul./ago. 2009.

BRASIL. Agência Nacional de Saúde Suplementar. Associação Médica Brasileira. Primeiras diretrizes clínicas na saúde suplementar - versão preliminar. Rio de Janeiro: ANS, 2009.

BRASIL. Ministério da Saúde. Secretaria Nacional de Assistência à Saúde. Instituto Nacional de Câncer. Coordenação
Nacional de Controle do Tabagismo e Prevenção Primária do Câncer. Abordagem e tratamento do fumante Consenso 2001. Rio de Janeiro: INCA, 2001.

Instituto Nacional do Câncer. Estimativas da incidência e mortalidade por câncer no Brasil. Rio de Janeiro: Ministério da Saúde, 2003.

Programa Nacional de Controle do Tabagismo e Outros Fatores de Risco de Câncer - Modelo Lógico e Avaliação. Rio de Janeiro: Ministério da Saúde, 2003.

. Secretaria de Vigilância em Saúde. Instituto Nacional de Câncer. Inquérito domiciliar sobre comportamentos de risco e morbidade referida de doenças e agravos não transmissíveis. Rio de Janeiro: Ministério da Saúde, 2004.

. Secretaria de Atenção à Saúde. Instituto Nacional de Câncer. Coordenação de Prevenção e Vigilância. Deixando de fumar sem mistérios: manual do coordenador. Rio de Janeiro: INCA, 2008.

. Ministério da Saúde. Instituto Nacional do Câncer. Programa de controle do tabagismo. Disponível em: $<$ www. inca.gov.br> Acesso em 04 maio 2010.

BURNS, D. M. Dependência de nicotina. In WILSON, BRAUNWALD, ISSELBACHER, ROOT, (Eds.). Harrison princípios de medicina interna.17. ed. Rio de Janeiro: McGraw-Hill do Brasil; Interamericana, 2008, p. 2726-39.

CARVAlHO, A. A.; GOMES, L.; LOUREIRO, A. M. L. Tabagismo em idosos internados em instituições de longa permanência. J Bras Pneumol, São Paulo, v. 36, n. 3, p. 339-46, maio/jun. 2010.

CARVALHO, C. R. S. O Instituto Nacional do Câncer e o controle do tabagismo: uma análise da gestão federal do tratamento do tabagismo no SUS. 2009 Dissertação (Mestrado na Modalidade Profissional de Saúde Pública) - Escola Nacional de Saúde Pública, Fundação Oswaldo Cruz, Rio de Janeiro, 2009. Disponível em: $<$ http://bvssp.icict.fiocruz.br/pdf/Carvalhocrsm.pdf $>$. Acesso em: 12 ago. 2010.

CAVALCANTE, T. M. O controle do tabagismo no Brasil: avanços e desafios. Rev. Psiquiatr. Clín., São Paulo, v. 32, n. 5, p. 283-300, set./out. 2005.

COELHO, A. C. et al. Conhecimentos sobre tabagismo entre estudantes de medicina. Rev. HCPA, Porto Alegre, v. 30, n. 2, p. 94-9, 2010.

FAGERSTROM, K. O. Measuring degree of physical dependence to tobacco smoking with reference to individualization of treatment. Addict Behav. v. 3, n. 4, p. 235-41, 1978.

FILHO, F. S. L. et al. Levantamento randomizado sobre a prevalência de tabagismo nos maiores municípios do Brasil. J Bras Pneumol, São Paulo, v. 35, n. 12, p. 1204-11, dez. 2009. 
HALTY, L. S. et al. Análise da utilização do Questionário de Tolerância de Fagerström (QTF) como instrumento de medida da dependência nicotínica. J Pneumol., São Paulo, v. 28, n. 2, p. 180-86, mar./abr. 2002.

HORTENSE, F. T. P; CARMAGNANI, M. I. S; BRÊTAS, A. C. P. O significado do tabagismo no contexto do câncer de laringe. Rev. Bras. Enferm., Brasília, v. 61. n. 1, p. 24-30, jan./fev. 2008.

IGLESIAS, R. J. P. et al. Controle do Tabagismo no Brasil. Washington: Banco Mundial, 2007. 136p.

INSTITUTO BRASILEIRO DE GEOGRAFIA E ESTATÍSTICA (IBGE). Ministério do Planejamento, Orçamento e Gestão. Diretoria de Pesquisas. Coordenação de Trabalho e Rendimento. Pesquisa Nacional de Amostra de Domicílios Tabagismo 2008. Rio de Janeiro: IBGE, 2009.

MARQUES, A. C. P. R.; RIBEIRO, M. Abuso e dependência da nicotina. Associação Brasileira de Psiquiatria. Projeto Diretrizes, 2002.

MUNDIM, M. M.; BUENO, G. N. Análise comportamental em um caso de dependência à nicotina. Rev. Bras. Ter. Comport. Cogn., São Paulo, v. 8, n. 2, p. 179-91, dez. 2006.

ORGANIZAÇÃO MUNDIAL DE SAÚDE (OMS). Classificação Estatística Internacional de Doenças e Problemas Relacionados à Saúde - Décima Revisão- (CID 10). Traduzido pela Faculdade de Saúde Pública de São Paulo - Centro Colaborador da OMS para Classificação de Doenças em Português. São Paulo: Editora da Universidade de São Paulo, 1997.

PAN AMERICAN ORGANIZATION. Smoking and health in the Americas: a 1992 report of the Surgeon General, in collaboration with the Pan American Health Organization. Atlanta (GE): PAHO, 1992.

PINTO, M.; UGÁ, M. A. D. Os custos de doenças tabaco-relacionadas para o Sistema Único de Saúde. Cad. Saúde Pública., Rio de Janeiro, v. 26, n. 6, p. 1234-45, jun. 2010.

PLANETA, C. S.; CRUZ, F. C. Bases neurofisiológicas da dependência do tabaco. Rev. Psiquiatr. Clín., São Paulo, v. 32, n. 5, p. 251-58, set./out. 2005.

PRESMAN, S.; CARNEIRO, E.; GIGLIOTTI, A. Tratamentos não-farmacológicos para o tabagismo. Rev. Psiquiatr. Clín., São Paulo, v. 32, n. 5, p. 267-75, set./out. 2005.

PROJETO ITC (Outubro 2009). ITC Brasil - resumo. Universidade de Waterloo, Waterloo, Ontário, Canadá; e Instituto Nacional de Câncer (INCA)/Ministério da Saúde. Brasil, 2009.

REICHERT, J. et al. Diretrizes para cessação do tabagismo. J Bras Pneumol., São Paulo, v. 34, n. 10, p. 845-80, out. 2008.

WORLD HEATH ORGANIZATION (WHO). Making a difference. World heath report. Geneva: WHO, 1999.

WHO report on the global tobacco epidemic, 2008: the MPOWER packaged. Geneva: WHO, 2008.
Anexo 1
Questionário
1) $\mathrm{O}$ (a) senhor(a) fuma?
( ) $\operatorname{sim}$ ( ) não
2) $\mathrm{O}$ (a) senhor(a) já fumou anteriormente?
( ) $\operatorname{sim} \quad$ ( ) não

3) Há quanto tempo o(a) senhor(a) fuma?
4) $O$ (a) senhor(a) pretende parar de fumar?
( ) $\operatorname{sim}$
( ) não
5) Gênero
( ) feminino ( ) masculino
6) Quantos anos o(a) senhor(a) tem?

7) Até que série o(a) senhor(a) estudou?

8) Qual a renda mensal de sua família?

( ) menos que 2 salários-mínimos

( ) de 2 a 5 salários-mínimos

( ) mais que 5 salários-mínimos 
Anexo 2

Nicotina

Teste de Fagerström para Dependência de

1) Quanto tempo depois de acordar você fuma o primeiro cigarro?
( ) dentro de 5 minutos
( ) entre 6 e 30 minutos
( ) entre 31 e 60 minutos
( ) após 60 minutos

2) Você acha difícil não fumar em locais onde o fumo é proibido (como igrejas, bibliotecas, etc.)?
( ) $\mathrm{Sim}$
( ) Não

3) Qual o cigarro do dia que traz mais satisfação (ou que mais detestaria deixar de fumar)?
( ) o primeiro da manhã
( ) outros

4) Quantos cigarros você fuma por dia?
( ) 10 ou menos
( ) 11 a 20
( ) 21 a 30
( ) 31 ou mais

5) Você fuma mais frequentemente pela manhã (ou nas primeiras horas do dia) que no resto do dia?
( ) $\operatorname{Sim}$
( ) Não

6) Você fuma mesmo quando está doente que precisa ficar de cama a maior parte do tempo?
( ) $\mathrm{Sim}$
( ) Não

Total:

Conclusão quanto ao grau de dependência:

0 a 2 pontos - muito baixo

3 a 4 pontos - baixo

5 pontos - médio

6 a 7 pontos - elevado

8 a 10 pontos - muito elevado 